\title{
Neoadjuvant Immunotherapy-Based Systemic Treatment in MMR-Deficient or MSI-High Rectal Cancer: Case Series
}

Rahel Demisse, $\mathrm{MD}^{1,2}$; Neha Damle, MD; Edward Kim, MD, PhD; Jun Gong, MD³; Marwan Fakih, MD4; Cathy Eng, MD;

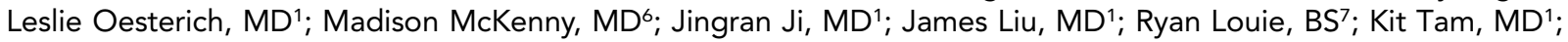
Sepideh Gholami, MD; Wissam Halabi, MD; Arta Monjazeb, MD, PhD ${ }^{10}$; Farshid Dayyani, MD ${ }^{11, *}$; and May Cho, MD ${ }^{1, \star}$

\begin{abstract}
Treatment options for locally advanced rectal cancer have continued to consist largely of chemotherapy, chemoradiation, and/or surgical resection. For patients who are unable to undergo these therapeutic modalities or who do not to experience a response to them, treatment options are limited. We report 3 cases of mismatch repair-deficient (dMMR) locally advanced adenocarcinoma of the rectum that showed significant response with neoadjuvant immunotherapy-based systemic treatment. The first patient was not eligible for standard therapy because of a history of radiotherapy to the prostate with concurrent comorbidities and therefore received single-agent pembrolizumab. The second patient did not respond to total neoadjuvant chemoradiation and subsequently received combined nivolumab and ipilimumab. The third patient had a known family history of Lynch syndrome and presented with locally advanced rectal cancer and a baseline carcinoembryonic antigen level of 1,566 ng/mL. She was treated using neoadjuvant pembrolizumab and FOLFOX (folinic acid, fluorouracil, oxaliplatin). In this small series, we suggest that single-agent and combined-modality neoadjuvant immunotherapy/chemotherapy appear to be safe and effective treatment options for patients with (dMMR) locally advanced rectal cancer. Our findings encourage further studies to investigate the role of neoadjuvant immunotherapy as a viable treatment strategy in this population.
\end{abstract}

J Natl Compr Canc Netw 2020;18(7):798-804 doi: $10.6004 /$ jnccn.2020.7558

\footnotetext{
${ }^{1}$ UC Davis Comprehensive Cancer Center, Sacramento, California; ${ }^{2}$ Loma Linda University Health, Loma Linda Medical Center, Loma Linda, California; ${ }^{3}$ Samuel Oschin Comprehensive Cancer Institute, Cedars-Sinai Health System, Los Angeles, California; ${ }^{4}$ City of Hope National Medical Center, Duarte, California; ${ }^{5}$ Vanderbilt-Ingram Cancer Center, Nashville, Tennessee; ${ }^{6}$ Department of Diagnostic Radiology, UC Davis Medical Center, Sacramento, California; ${ }^{7}$ UC Davis Medical Center, California; ${ }^{8}$ Department of Surgical Oncology and ${ }^{9}$ Department of Colorectal Surgery, UC Davis Medical Center, Sacramento, California; ${ }^{10}$ Department of Radiation Oncology, UC Davis Comprehensive Cancer Center, Sacramento, California; and ${ }^{11}$ Division of Hematology/ Oncology, Department of Medicine, University of California Irvine, Orange, California.
}

*These authors contributed equally.
In 2020, 43,340 new cases of rectal cancer were diagnosed, most of which were localized or locoregional disease. ${ }^{1}$ It is estimated that $10 \%$ to $15 \%$ of all sporadic colorectal cancer (CRC) cases are mismatch repair-deficient (dMMR) or microsatellite instability-high (MSI-H) ${ }^{2,3}$ The immune checkpoint inhibitors pembrolizumab, nivolumab, and combination nivolumab/ipilimumab have been approved in dMMR or MSI-H metastatic CRC that is refractory or intolerant to standard therapies. ${ }^{4-7}$ However, the standard of care for locally advanced rectal cancer remains chemoradiation, systemic chemotherapy, and, commonly, surgical resection. For patients who are unable to undergo these therapeutic modalities or who do not to experience a response to them, treatment options remain limited. This report presents 3 patients with dMMR locally advanced adenocarcinoma of the rectum who experienced significant response to single-agent immunotherapy or combination neoadjuvant immuno/chemotherapy, and briefly reviews the literature on neoadjuvant therapy in locally advanced CRC and the benefit of immunotherapy in CRC with dMMR.

\section{Case Presentations}

Case 1

A man aged 81 years with a history of prostate cancer (diagnosed 10 years ago, status post definitive brachytherapy) presented with rectal bleeding. His workup, including colonoscopy, revealed 2 large masses in the rectum with central ulceration. Pathology was significant for moderately to poorly differentiated invasive adenocarcinoma with loss of MSH2 and MSH6 expression in both tumors. An MRI of the pelvis showed a synchronous T3cN1 tumor in the low rectum and a T1/T2N0 tumor in the midrectum. The mesorectal fascia was threatened, and an extramesorectal/left internal iliac lymph node was suspicious for tumor involvement. Given his history of radiation for prostate cancer and the presence of baseline neuropathy, he was a poor candidate for neoadjuvant 
chemoradiation or neoadjuvant FOLFOX (folinic acid, fluorouracil, oxaliplatin). After an in-depth discussion with regard to risk, benefit, and available data on immunotherapy, the patient was started on neoadjuvant pembrolizumab, $200 \mathrm{mg}$ every 3 weeks.

After completing 3 cycles of pembrolizumab, restaging MRI showed tumor regression of the low rectal lesion from 5 to $2.5 \mathrm{~cm}$ (tumor regression grade 3, $>50 \%$ fibrosis). The tumor no longer seemed to involve the intersphincteric fat or external sphincter. In addition, the tumor had retracted away from the mesorectal margin with resolution of associated adenopathy. The midrectal tumor had also resolved with no distinct residual tumor signal (Figures 1-3).

The patient continued to have partial response on subsequent imaging, and tolerated, in total, 11 cycles of pembrolizumab. However, he developed grade 2 fatigue attributed to immunotherapy, which led to therapy discontinuation. The patient was off of pembrolizumab for 6 months without disease progression. He subsequently underwent biopsy of the low rectal lesion to assess for residual disease versus necrotic tissue. Pathology evaluation did not show any evidence of active malignancy supporting complete response to pembrolizumab. At the time of this report, he has been observed for 17 months without any evidence of disease recurrence.

\section{Case 2}

The second patient was a man aged 55 years with a history of stage II, right-sided colon cancer diagnosed in 1993. Shortly after diagnosis, his course was complicated by perforation and he underwent a hemicolectomy followed by adjuvant 5-FU. In September 2018, he presented with rectal pain and underwent diagnostic flexible sigmoidoscopy. This revealed a $2.5-\mathrm{cm}$ rectal mass proximal to the anal verge. Pathology findings from a biopsy were significant for infiltrating colonic adenocarcinoma with loss of nuclear expression of PMS2. The combined positive score for PD-L1 expression was $10 \%$. Next-generation sequencing showed a tumor mutational burden of 21.3 mutations per megabase. Additional nextgeneration sequencing identified potentially actionable mutations in SETD2, APC, FBXW7, MSH3, ATM, and $B R C A 1$. Other biologically relevant mutations were also seen in TCF7L2, KMT2C, CTCF, MEN1, NBN, MSH6, PMS2, PAX5, and GRIN2A. A staging MRI of the pelvis showed a $3.0-\mathrm{cm}$ lower rectal mass, T3cN1, with threatened mesorectal fascia. A CT of the chest, abdomen, and pelvis did not reveal evidence of metastatic disease. The patient was started on total neoadjuvant therapy and received 8 cycles of FOLFOX followed by concurrent chemoradiation with oral capecitabine at $1,650 \mathrm{mg}$ twice a day. He tolerated this therapy well with the exception of grade 2 neuropathy. Unfortunately, a restaging MRI 6 weeks after completion of concurrent chemoradiation revealed a persistent $3-\mathrm{cm}$ rectal tumor with threatened mesorectal fascia. This tumor was essentially unchanged in size when compared with pretreatment imaging. He was evaluated for colorectal surgery and considered a poor candidate due to inability to safely achieve a good surgical margin.

Given the lack of response to total neoadjuvant therapy and ongoing threatened mesorectal margin, the patient was started on neoadjuvant immunotherapy with nivolu$\mathrm{mab}$ at $3 \mathrm{mg} / \mathrm{kg}$ and ipilimumab at $1 \mathrm{mg} / \mathrm{kg}$ intravenously every 3 weeks. The patient tolerated treatment well, experiencing only minor grade 1 fatigue and rash.

A

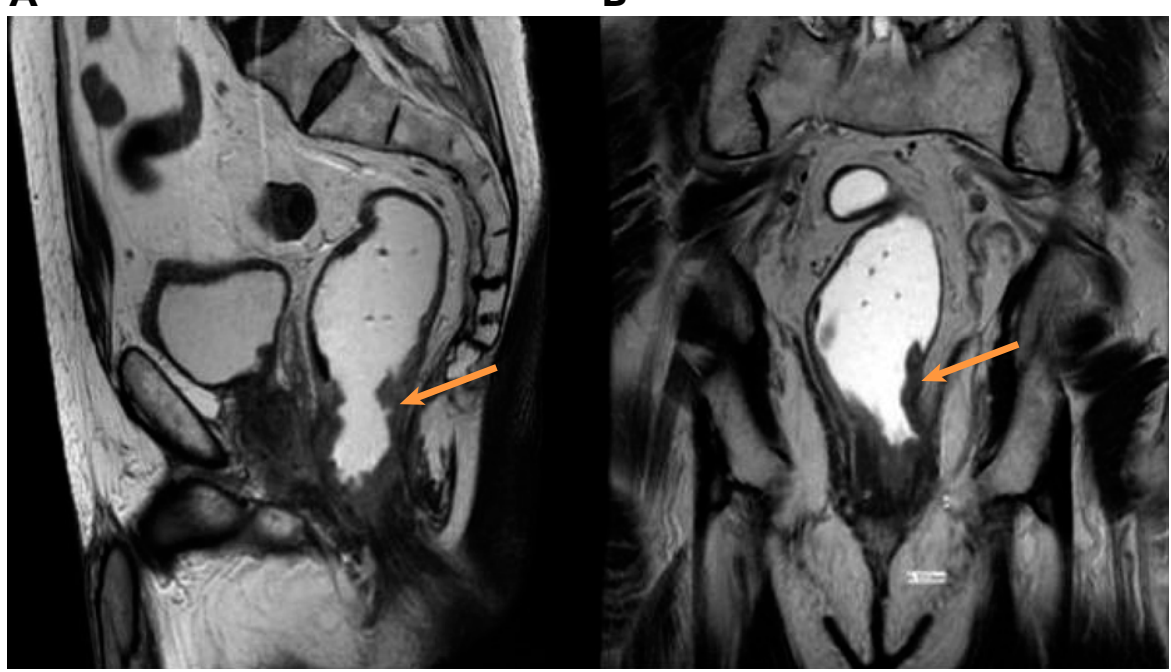

Figure 1. Baseline imaging for Case 1. (A) Sagittal and (B) coronal T2-weighted images through rectum after administration of rectal gel show a 5.3-cm mass along the lower rectum, T3cNO. Mesorectal fascia is threatened (not seen here). 
A

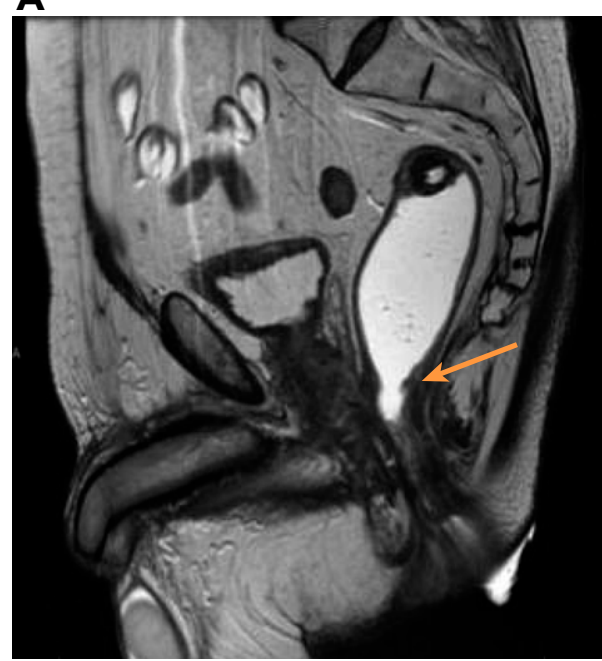

B

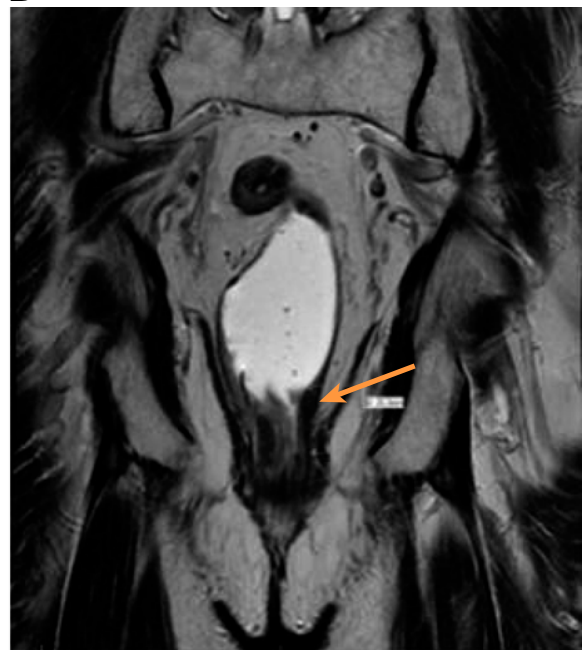

Figure 2. Case 1 after 6 cycles of pembrolizumab. (A) Sagittal and (B) coronal T2-weighted images through rectum after administration of rectal gel after treatment showing interval reduction in tumor size when compared with prior examination. Although not seen in its entirety on these representative images, the mass had $>50 \%$ fibrosis, compatible with tumor regression grade 3 .

A restaging MRI after 3 cycles of treatment showed dense fibrosis with grade 2 tumor regression and no obvious residual tumor. The mesorectal fascia was no longer threatened, and there was resolution of the adenopathy (Figures 4 and 5). Colonoscopy showed a $1-\mathrm{cm}$ friable ulcer at the anal verge. Extensive biopsies were performed of this section, and pathology showed ulceration with acute inflammation and granulation tissue, with no evidence of residual tumor. The patient decided to forgo definitive surgery and continue maintenance nivolumab therapy along with close surveillance. After completing 7 cycles of maintenance nivolumab, follow-up imaging in January 2020 showed a $3.1-\mathrm{cm}$ low rectal tumor. However, repeat biopsy of this mass again showed necrosis and inflammation, confirming a sustained complete response (CR) for 12 months as of the current writing.

\section{Case 3}

The third patient was a woman aged 38 years with a known family history of Lynch syndrome. She initially presented with bright red blood per rectum over several months without associated pain or change in bowel habits. She underwent colonoscopy, which showed a
A

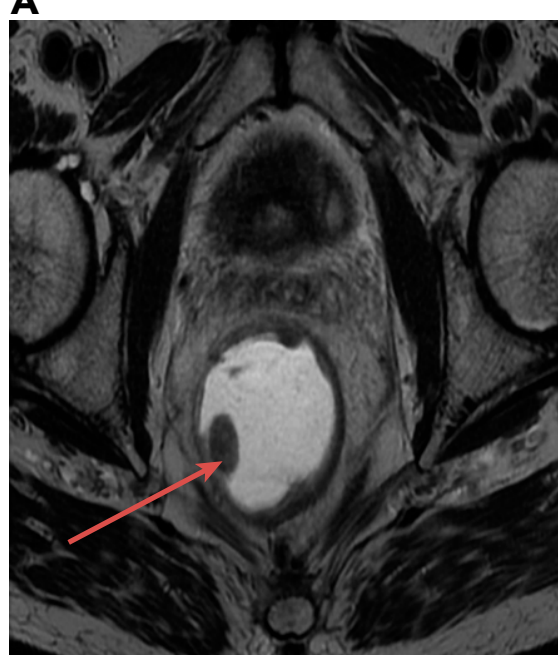

B

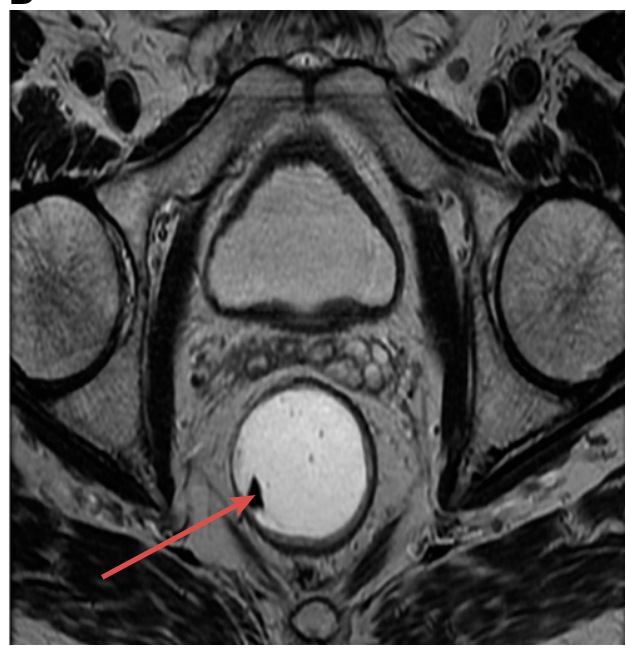

Figure 3. (A) Baseline axial T2-weighted image after administration of rectal gel in Case 1, with a polypoid mass seen at approximately 8:00. (B) After 6 cycles of pembrolizumab, axial T2-weighted image after administration of rectal gel at the level of previously seen polypoid mass shows no residual mass, compatible with tumor regression grade 1 . 
A

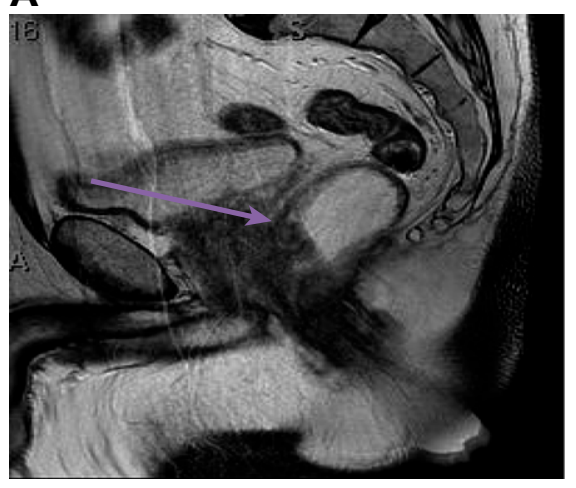

B

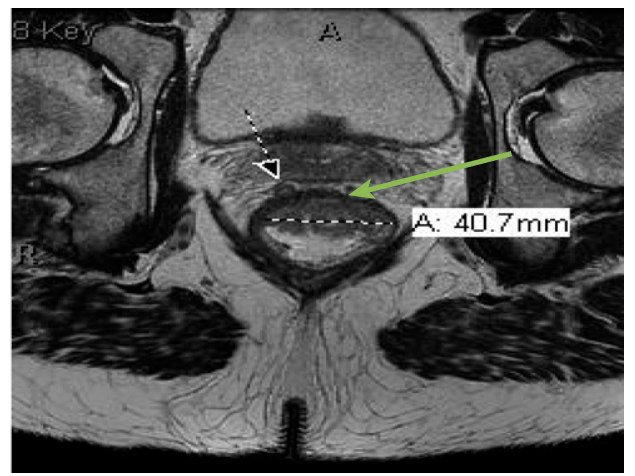

Figure 4. Baseline imaging for Case 2 showing circumferential 3.0-cm ( $A$; craniocaudal, purple arrow) and 4.1-cm mass ( $B$; mediolateral, green arrow) located $4.5 \mathrm{~cm}$ from anal verge. Mesorectal fascia is threatened. Tumor is T3cN1 (not seen here).

fungating, partially obstructing tumor at 10 to $14 \mathrm{~cm}$ from the anal verge. Pathology findings were consistent with invasive moderately differentiated adenocarcinoma with mucinous features. Immunohistochemistry staining for MMR proteins showed loss of expression of MSH2 but intact expression of MLH1, PMS2, and MSH6. MRI staging showed a diffuse, lobulated, and mucinousseeming tumor involving the midrectum, $10.3 \mathrm{~cm}$ from the anal verge. The tumor straddled the anterior peritoneal reflection and invaded the mesorectal fat; no sphincter involvement was seen. The mesorectal fascia was involved with tumor within $1 \mathrm{~mm}$. A $\mathrm{CT}$ of the chest and abdomen did not show any metastases. However, her CEA level was elevated at $1,566.2 \mathrm{ng} / \mathrm{mL}$ before treatment. Thus the patient was staged as having clinical T3N2M0, or stage IIIB, rectal cancer.

Because the patient had an MSI-H tumor (hereditary nonpolyposis CRC) and high-risk disease (significantly elevated CEA level and N2 disease), she was offered pembrolizumab in addition to standard chemotherapy with FOLFOX. This decision was made in light of data from stage IV disease showing a higher likelihood of durable response with the addition of immunotherapy. ${ }^{8}$

With the exception of a minor grade 1 rash, the patient tolerated 7 cycles of FOLFOX with concurrent pembrolizumab well. Given this excellent response, she opted out of chemoradiation. the available posttreatment imaging was performed at an outside facility and was of poor quality. Given the limitations regarding radiographic data, her overall response to therapy was assessed based on surgical pathology. She underwent a robotic-assisted laparoscopic low anterior resection with transvaginal extraction and side-to-end colorectal anastomosis, hysterectomy, and oophorectomy. All submitted pathologic specimens, including 16 lymph nodes, were negative for carcinoma, hence showing a pathologic CR (pCR; ypTOpN0). At the
A

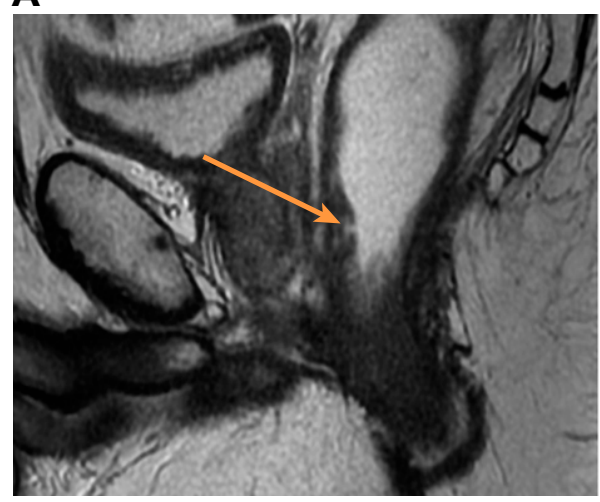

B

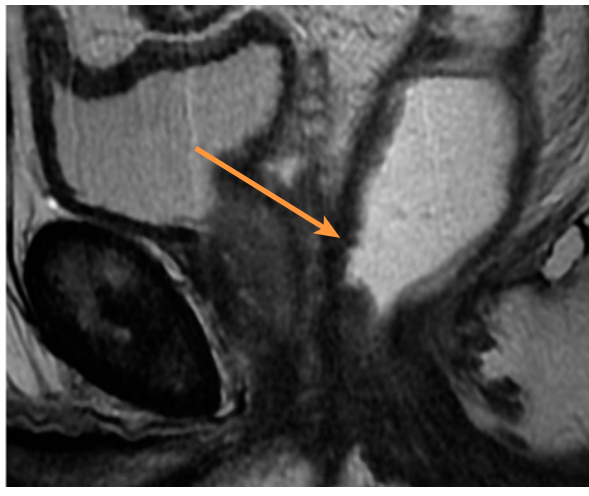

Figure 5. (A) After treatment with total neoadjuvant therapy, sagittal T2-weighted image of rectum after administration of rectal gel shows rectal mass seen along anterior rectal wall in Case 2. Not entirely seen here, however, is that overall imaging showed stable disease-a 3-cm low rectal tumor after total neoadjuvant therapy, T3cN1 with a threatened mesorectal fascia. (B) After 3 cycles of nivolumab and ipilimumab, sagittal T2-weighted image of rectum after administration of rectal gel shows interval reduction in size of previously seen tumor. No obvious residual tumor, TONO with mesorectal fascia that is no longer threatened (not seen here). 
time of writing, this patient had been observed for 10 months without disease recurrence.

\section{Discussion}

Rectal cancers with dMMR or MSI-H have been linked to a poorer prognosis and resistance to conventional therapies. ${ }^{9}$ Cytotoxic chemotherapy including fluoropyrimidine-based regimens is widely used in CRC. ${ }^{10}$ However, current evidence supports that tumors with dMMR or MSI-H are resistant to conventional chemotherapy, mainly because of the inability of the cells to detect mismatched and unpaired bases..$^{10-12}$ This leads to tolerance of DNA damage and allows cells to defer apoptosis. ${ }^{11}$ For locally advanced rectal cancer, combined modality therapy with concurrent chemoradiation and surgery is the mainstay of treatment. ${ }^{13,14}$ However, a recent retrospective study using the National Cancer Database showed that microsatellite instability was independently associated with a reduction in pCR after completion of neoadjuvant chemoradiation in patients with locally advanced rectal cancer. ${ }^{2}$ In the study, all patients were treated using definitive chemoradiation (median dose of $50.4 \mathrm{~Gy}$ ) followed by resection. The overall pCR rate was $8.6 \%$, including $8.9 \%$ for mismatch repair-proficient (pMMR) tumors and $5.9 \%$ for dMMR tumors. ${ }^{2}$ Notably, most patients had T3 (85\%) or node-positive (59\%) disease. In another recent study, total neoadjuvant therapy showed pCR in 43 of 235 patients (18\%) who received total neoadjuvant therapy followed by surgery; no data were reported on the response rate in patients with dMMR/MSI-H tumors. ${ }^{15}$

In the chemorefractory patient population, immunotherapy has afforded promising and durable responses. ${ }^{7}$ Le et $\mathrm{al}^{16}$ initially evaluated the role of PD-1 blockade (pembrolizumab) across 11 tumor types with dMMR in
KEYNOTE-016. In the study, patients with refractory metastatic dMMR CRCs had an immune-related objective response rate of $40 \%$ compared with $0 \%$ in pMMR CRCs. This led to the first tissue-agnostic FDA approval in 2017 for pembrolizumab. A subsequent multicohort phase II trial (CheckMate-142) ${ }^{6}$ evaluated nivolumab or nivolumab combinations in recurrent and metastatic MSI-H and non-MSI-H colon cancer. In the single-agent nivolumab cohort, 23 of 74 patients with heavily pretreated dMMR/MSI-H CRC achieved an investigator-assessed objective response of $31.1 \%$ after treatment with nivolumab. Fifty-one patients (69\%) had disease control for $\geq 12$ weeks. Durable response lasting $\geq 12$ months was seen in 8 of the 23 patients, and median duration of response was not yet reached at the time of publication of CheckMate-142. ${ }^{6}$ The nivolumab and ipilimumab cohort of CheckMate-142 also revealed an investigator-assessed overall response rate of 55\% at a median follow-up of 13.4 months in previously treated patients; surprisingly, $3 \%$ of patients achieved CR. ${ }^{7}$ The 12-month progression-free and overall survival rates were $71 \%$ and $85 \%$, respectively, and median duration of response was not reached. ${ }^{7}$ Notably, a recent update revealed that the CR rate increased from $3 \%$ to $6 \%$ with long-term follow-up. ${ }^{17}$ New data from a report out of the third cohort of CheckMate-142 showed an objective response rate of $60 \%$ and a $7 \%$ CR in 45 patients with no prior treatment of MSI-H metastatic CRC. ${ }^{8}$

To date, clinical trials have focused on assessing the benefits of immunotherapy in the metastatic setting only. Recently, the role of immunotherapy was further explored in the neoadjuvant setting by Chalabi et $\mathrm{al}^{18}$ in the NICHE study. They conducted a phase II noncompetitive study that evaluated a short course of neoadjuvant

Table 1. Response Rates From Selected Trials in Colorectal Cancer

\begin{tabular}{|c|c|c|c|c|c|}
\hline Trial & $\begin{array}{l}\text { Cancer Stage/ } \\
\text { Line of Treatment }\end{array}$ & $\mathbf{n}$ & Regimen & $\begin{array}{l}\text { PCR } \\
(\%)\end{array}$ & $\begin{array}{l}\text { dMMR ORR } \\
(\%)\end{array}$ \\
\hline Bosset et al ${ }^{14}$ & III & 1,011 & $\begin{array}{l}\text { RT alone } \\
\text { vs } \\
\text { ChemoRT }\end{array}$ & $\begin{array}{c}5.3 \\
13.7\end{array}$ & - \\
\hline Cercek et $\mathrm{al}^{15}$ & $\|/\| I I$ & 811 & $\begin{array}{c}\text { Neoadjuvant chemoRT + adjuvant therapy } \\
\text { vs } \\
\text { TNT }\end{array}$ & $\begin{array}{l}17 \\
18\end{array}$ & - \\
\hline Chalabi et al ${ }^{18}$ & I-III & 14 & Nivolumab + ipilimumab & 57 (dMMR) & - \\
\hline Lenz et $\left.a\right|^{8}$ & $\begin{array}{l}\text { IV } \\
\text { 1st line }\end{array}$ & 45 & Nivolumab + ipilimumab & - & 60 \\
\hline Le et $\mathrm{al}^{16}$ & $\begin{array}{c}\text { IV } \\
\geq 2 \text { nd line }\end{array}$ & 41 & Pembrolizumab & - & 40 \\
\hline Overman et $\mathrm{al}^{7}$ & $\begin{array}{c}\text { IV } \\
\geq 2 \text { nd line }\end{array}$ & 119 & Nivolumab + ipilimumab & - & 55 \\
\hline Overman et $a^{6}$ & $\begin{array}{c}\text { IV } \\
\geq 2 \text { nd line }\end{array}$ & 74 & Nivolumab & - & 31.1 \\
\hline
\end{tabular}

Abbreviations: chemoRT, chemoradiation; dMMR, mismatch repair-deficient; ORR, overall response rate; $\mathrm{PCR}$, pathologic complete response; RT, radiotherapy; TNT, total neoadjuvant therapy. 
ipilimumab + nivolumab in early-stage dMMR and pMMR colon cancer. Results showed major pathologic response (defined as $<5 \%$ viable tumor cells) in $100 \%$ of patients with dMMR tumors. Of the 7 patients with dMMR tumors, 4 achieved pCR; however, none of the pMMR tumors had major pathologic responses. ${ }^{18}$ Table 1 summarizes response rates from selected trials in CRC.

In light of the aforementioned recent data from the NICHE study, ${ }^{18}$ our patient in Case 1 received neoadjuvant therapy using single-agent pembrolizumab and showed a robust partial response on imaging. This patient had not experienced any disease progression 6 months posttreatment, suggesting that the residual disease seen on imaging likely represented necrotic tissue. This result was also in line with a recently published article by Zhang et al ${ }^{19}$ showing CR in 2 patients with locally advanced dMMR/MSI-H rectal cancer after treatment with neoadjuvant nivolumab. The patient in Case 2 minimal response to neoadjuvant chemotherapy. He then underwent neoadjuvant immunotherapy and subsequently achieved a satisfactory response on MRI and a biopsy showing pCR. This result is consistent with those of the recent phase II NICHE study, ${ }^{18}$ which showed the efficacy of neoadjuvant immunotherapy with nivolumab + ipilimumab in patients with early-stage dMMR colon cancer. In Case 3, pCR and normalization of tumor markers was achieved using neoadjuvant concurrent chemotherapy and immunotherapy in a patient with known Lynch syndrome; the patient tolerated the combination safely. This case showed that pCR could be achieved in patients with dMMR rectal cancer without the use of radiation therapy. Such an opportunity may be particularly important for female patients with reproductive potential, as long as follow-up data reveal comparable risk for local recurrence. This result supports the literature showing that patients with dMMR tumors may benefit from first-line treatment using immunotherapy, either stand-alone or in combination with chemotherapy. ${ }^{8,18,20}$ Ongoing phase III studies are comparing immunotherapy alone with pembrolizumab versus a combination of immunotherapy and chemotherapy versus investigator-choice chemotherapy and modified FOLFOX/bevacizumab combination chemotherapy with or without atezolizumab or atezolizumab monotherapy in the first-line setting for metastatic dMMR CRC. ${ }^{21,22}$

\section{Conclusions}

In this small series, treatment of locally advanced dMMR rectal cancer using immunotherapy either as a single agent or in combination with chemotherapy led to significant response. We showed that neoadjuvant therapy using a single-agent PD-1 inhibitor, combined PD-1/CTLA-4 inhibitors, or PD-1 inhibitor plus FOLFOX combination can be a safe and effective treatment option in selected patients with locally advanced dMMR rectal cancer. These cases provide encouraging evidence for further studies to investigate the role of neoadjuvant immunotherapy in all patients with locally advanced or metastatic dMMR rectal cancer.

Submitted December 5, 2019; accepted for publication March 6, 2020.

Disclosures: Dr. Kim has disclosed that he receives grant/research support from Celgene, Astellas, Samumed, Boston Biomedical, Halozyme, EpicentRx Merck, Oncomed, and Bristol-Myers Squibb; and receives consulting fees from Lilly and Celgene. Dr. Gong has disclosed that he receives consulting fees/ honoraria from Amgen and Astellas. Dr. Fakih has disclosed that he receives consulting fees from Array, Amgen, Pfizer Inc., and Bayer; serves on the speaker's bureau of Amgen and Guardant360; and receives grant/research support from AstraZeneca, Amgen, and Novartis. Dr. Monjazeb has disclosed that he is a scientific advisor for Incyte, Dynavax, and AstraZeneca, and receives grant/research support from Genentech, Merck, Incyte, EMD Serono, Transgene, and Bristol-Myers Squibb. Dr. Dayyani has disclosed that he receives consulting fees from Genentech, Eisai, Exelixis, Array, and Foundation Medicine; serves on the speaker's bureaus of Amgen, Genentech, Exelixis, Eisai, and Sirtex; and receives grant/research support from Bristol-Myers Squibb, Merck, AstraZeneca, and Taiho. Dr. Cho has disclosed that she receives consulting fees/honoraria from Amgen, Taiho, Astella, Ipsen, Eisai, Exelixis, and Incyte. The remaining authors have disclosed that they have not received any financial consideration from any person or organization to support the preparation, analysis, results, or discussion of this article.

Correspondence: Rahel Demisse, MD, UC Davis Comprehensive Cancer Center, 4501 X Street, Sacramento, CA 95817. Email: rzdemisse@gmail.com

\section{References}

1. Siegel RL, Miller KD, Jemal A. Cancer statistics, 2020. CA Cancer J Clin 2020;70:7-30

2. Hasan S, Renz P, Wegner RE, et al. Microsatellite instability (MSI) as an independent predictor of pathologic complete response (PCR) in locally advanced rectal cancer: a National Cancer Database (NCDB) analysis [published online September 13, 2018]. Ann Surg, doi: 10.1097/ SLA.0000000000003051

3. Koopman M, Kortman GA, Mekenkamp L, et al. Deficient mismatch repair system in patients with sporadic advanced colorectal cancer. Br J Cancer 2009;100:266-273.

4. Le DT, Kavan P, Kim TW, et al. Pembrolizumab for patients with advanced microsatellite instability high (MSI-H) colorectal cancer [abstract]. J Clin Oncol 2018;36(Suppl):Abstract 35414.

5. Marcus L, Lemery SJ, Keegan P, et al. FDA approval summary: pembrolizumab for the treatment of microsatellite instability-high solid tumors. Clin Cancer Res 2019;25:3753-3758.

6. Overman MJ, McDermott R, Leach JL, et al. Nivolumab in patients with metastatic DNA mismatch repair-deficient or microsatellite instability-high colorectal cancer (CheckMate 142): an open-label, multicentre, phase 2 study. Lancet Oncol 2017;18:1182-1191.

7. Overman MJ, Lonardi S, Wong KYM, et al. Durable clinical benefit with nivolumab plus ipilimumab in DNA mismatch repair-deficient/microsatellite instability-high metastatic colorectal cancer. J Clin Oncol 2018;36: 773-779.

8. Lenz HJ, van Cutsem E, Limon ML, et al. Durable clinical benefit with nivolumab (NIVO) plus low-dose ipilimumab (IPI) as first-line therapy in microsatellite instability-high/mismatch repair deficient (MSI-H/dMMR) metastatic colorectal cancer (mCRC) [abstract]. Ann Oncol 2018;29(Suppl 8): Abstract VIII714.

9. Samowitz WS, Curtin K, Wolff RK, et al. Microsatellite instability and survival in rectal cancer. Cancer Causes Control 2009;20: 1763-1768. 
10. Jo WS, Carethers JM. Chemotherapeutic implications in microsatellite unstable colorectal cancer. Cancer Biomark 2006;2:51-60.

11. Carethers JM, Smith EJ, Behling CA, et al. Use of 5-fluorouracil and survival in patients with microsatellite-unstable colorectal cancer. Gastroenterology 2004;126:394-401.

12. Sargent DJ, Marsoni S, Monges G, et al. Defective mismatch repair as a predictive marker for lack of efficacy of fluorouracil-based adjuvant therapy in colon cancer. J Clin Oncol 2010;28: 3219-3226.

13. Salem ME, Hartley M, Unger K, et al. Neoadjuvant combined-modality therapy for locally advanced rectal cancer and its future direction. Oncology (Williston Park) 2016;30:546-562.

14. Bosset JF, Calais G, Mineur L, et al. Enhanced tumorocidal effect of chemotherapy with preoperative radiotherapy for rectal Cancer: preliminary results-EORTC 22921. J Clin Oncol 2005;23:5620-5627.

15. Cercek A, Roxburgh CSD, Strombom P, et al. Adoption of total neoadjuvant therapy for locally advanced rectal cancer. JAMA Oncol 2018;4:e180071.

16. Le DT, Uram JN, Wang H, et al. PD-1 blockade in tumors with mismatchrepair deficiency. N Engl J Med 2015;372:2509-2520.

17. Overman MJ, Lonardi S, Wong KYM, et al. Nivolumab (NIVO) + low-dose ipilimumab (IPI) in previously treated patients (pts) with microsatellite instability-high/mismatch repair-deficient (MSI-H/dMMR) metastatic colorectal cancer (mCRC): long-term follow-up [abstract]. J Clin Oncol 2019; 37(Suppl):Abstract 635
18. Chalabi M, Fanchi LF, Van den Berg JG, et al. Neoadjuvant ipilimumab plus nivolumab in early stage colon cancer. Ann Oncol 2018;29(Suppl 8): Abstract VIII731.

19. Zhang J, Cai J, Deng Y, et al. Complete response in patients with locally advanced rectal cancer after neoadjuvant treatment with nivolumab. Oncolmmunology 2019;8:e1663108.

20. Sinicrope FA, Ou FS, Shi Q, et al. Randomized trial of FOLFOX alone or combined with atezolizumab in adjuvant therapy for patients with stage III colon cancer and deficient DNA mismatch repair or microsatellite instability (ATOMIC, Alliance A021502) [abstract]. J Clin Oncol 2017; 35(Suppl):Abstract TPS3630.

21. Diaz LA, Le DT, Yoshino T, et al. KEYNOTE-177: phase 3, open-label, randomized study of first-line pembrolizumab (Pembro) versus investigator-choice chemotherapy for mismatch repair-deficient (dMMR) or microsatellite instability-high (MSI-H) metastatic colorectal carcinoma (mCRC) [abstract]. J Clin Oncol 2018;36(Suppl):Abstract TPS877

22. Lee JJ, Yothers G, Jacobs SA, et al. Colorectal Cancer Metastatic dMMR Imuno-Therapy (COMMIT) study (NRG-GI004/SWOG-

S1610): a randomized phase III study of mFOLFOX6/bevacizumab combination chemotherapy with or without atezolizumab or atezolizumab monotherapy in the first-line treatment of patients (pts) with deficient DNA mismatch repair (dMMR) metastatic colorectal cancer (mCRC) [abstract]. J Clin Oncol 2019;37(Suppl):Abstract TPS728.

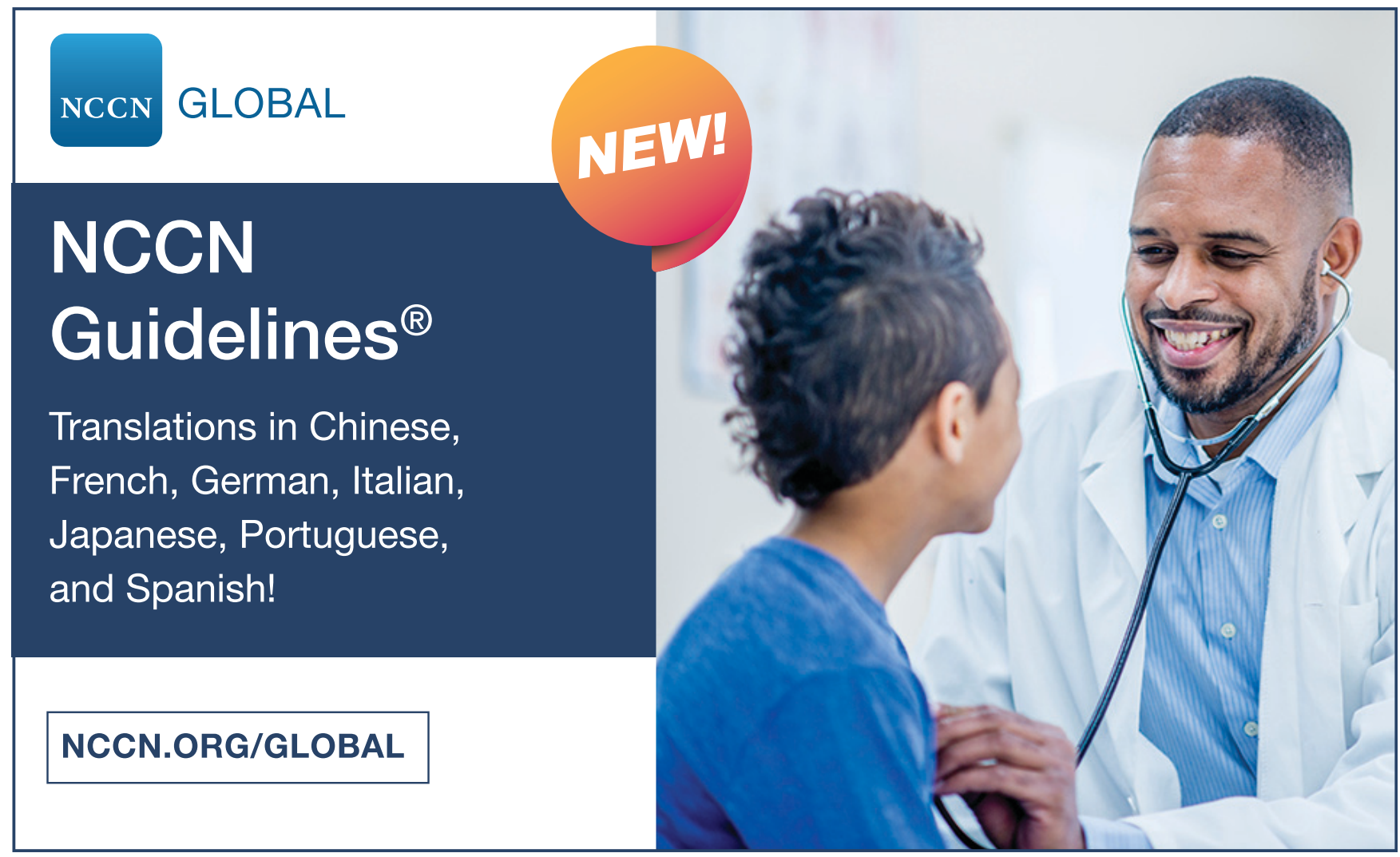

